\title{
Parametric form of QCD travelling waves
}

\author{
R. Peschansk:* \\ Service de physique théorique, CEA/Saclay, 91191 Gif-sur-Yvette cedex, France ${ }^{\dagger}$
}

\begin{abstract}
We derive parametric travelling-wave solutions of non-linear QCD equations. They describe the evolution towards saturation in the geometric scaling region. The method, based on an expansion in the inverse of the wave velocity, leads to a solvable hierarchy of differential equations. A universal parametric form of travelling waves emerges from the first two orders of the expansion.
\end{abstract}

1. Since the last years, progress is being made in the understanding of the QCD evolution in rapidity towards saturation, i.e. the high-density limit of weekly interacting partons probed at high energy. In particular, a link has been established [1] between travelling-wave solutions of non-linear equations and geometric scaling [2] of the gluon distribution function $\mathcal{N}(L, Y)$, where $L \equiv \log k^{2}$ is the transverse momentum variable and $Y$ the rapidity.

In the mean-field approximation we will consider here, $\mathcal{N}$ obeys [3] the nonlinear Balitsky-Kovchegov (BK) evolution equation

$$
\partial_{Y} \mathcal{N}=\chi\left(-\partial_{L}\right) \mathcal{N}-\mathcal{N}^{2}
$$

where, for convenience, rapidity $Y$ is measured in units of $\frac{\alpha_{s} N_{c}}{\pi}$ the strong coupling constant, kept fixed.

$$
\chi(\gamma)=2 \psi(1)-\psi(\gamma)-\psi(1-\gamma)
$$

is the Balitsky Fadin Kuraev Lipatov (BFKL) kernel [4].

The identification of Eq. (11), in the diffusive approximation of the kernel, with the Fisher and Kolmogorov-PetrovskyPiscounov (F-KPP) non-linear equation [5] infers [6] the existence of asymptotic travelling wave solutions in rapidity, see Fig [ The mechanism for the formation of travelling waves is more general [] and applies also [1] to the BK equation Eq.(11). Starting with the initial condition $\mathcal{N}\left(L, Y_{0}\right) \sim \exp \left(-\gamma_{0} L\right)$, travelling wave solutions $\mathcal{N}(L-c Y)$ are formed during the $Y$-evolution with the following features [7]:

- "pushed front": $\gamma_{0}<\gamma_{c} ; \quad c=\chi(\gamma) / \gamma$, with $\gamma=\gamma_{0}$

- "pulled front": $\gamma_{0} \geq \gamma_{c} ; \quad c=\chi(\gamma) / \gamma$, with $\gamma=\gamma_{c}$,

where, for the BK equation, $\gamma_{c}=.6275$ is the critical anomalous dimension, solution of $\chi\left(\gamma_{c}\right) / \gamma_{c}=\chi^{\prime}\left(\gamma_{c}\right)=c$, where $c=4.883$ is the critical velocity and $\gamma_{0}=1>\gamma_{c}$ (color transparency). For the F-KPP equation, the corresponding values are $\gamma_{c}=1$ and $c=2$. These powerful results come from a "locking" of the solutions of the linear part of the kernel due to the non-linearities. For the "pulled fronts", it has universal properties, i.e. the locking is independent of the form of non linearities and initial conditions. For the "pushed fronts" it is not so.

However, there are limitations of these solutions. They may require very large ranges in $Y$ and $L$ to appear. Moreover, while the wave structure is clear, the form of the front deduced from the linear kernel does not extend to the region where non-linear effects are important. In fact, only very few information on the non-linear term has been used. The goal of the present paper is to present a different method allowing to analytically derive travelling wave solutions taking into account the non-linear terms of the equation.

The values of $\gamma$ and $c$ coming from the knowledge of asymptotic properties are considered as input parameters for travelling wave solutions of the full non-linear equation, and one uses the fact, already noticed in the literature for the F-KPP equation [8], that $1 / c$ can be taken as a small expansion parameter of the full solution.

2. Let us start with Eq.(11), where the integro-differential operator $\chi\left(-\partial_{L}\right)$ will be defined with the help of a limited expansion around the value of $\gamma$ relevant for the travelling wave solutions, i.e., either $\gamma_{0}$ or $\gamma_{c}$ depending whether we are in a "pushed" or "pulled" front situation.

$$
\chi\left(-\partial_{L}\right)=\chi(\gamma) \mathbf{1}+\chi^{\prime}(\gamma)\left(-\partial_{L}-\gamma \mathbf{1}\right)+\frac{1}{2} \chi^{\prime \prime}(\gamma)\left(-\partial_{L}-\gamma \mathbf{1}\right)^{2}+\frac{1}{6} \chi^{(3)}(\gamma)\left(-\partial_{L}-\gamma \mathbf{1}\right)^{3}+\cdots .
$$

We shall consider truncations of (3) in the number of derivatives to some value $P$. We are looking for a solution in the vicinity of the selected anomalous dimension $\gamma$. For instance the diffusive approximation equivalent to the F-KPP equation is obtained with $P=2$. The validity of the approximation will be discussed.

\footnotetext{
† URA 2306, unité de recherche associée au CNRS.
} 
A generic travelling wave (i.e. geometric scaling) solution has the form $\mathcal{N}(z \equiv b L-a Y)$, with $a, b$ fixed parameters. Inserting it into Eq.(11), the partial differential BK equation (11) turns into an ordinary differential equation

$$
A_{0} \mathcal{N}-\mathcal{N}^{2}+\left(a-A_{1} b\right) \frac{d \mathcal{N}}{d z}+\sum_{p=2}^{P}(-1)^{p} A_{p} b^{p} \frac{d^{p} \mathcal{N}}{(d z)^{p}}=0
$$

where

$$
A_{p}=\sum_{i=0}^{P-p}(-1)^{i} \frac{\chi^{i+p}(\gamma)}{i !} \gamma^{i}
$$

With suitable redefinitions

$$
U \equiv \frac{\mathcal{N}}{A_{0}} ; \quad c \equiv \frac{a}{A_{0}}-\frac{A_{1}}{\sqrt{A_{0} A_{2}}} ; \quad s \equiv z / c ; \quad b \equiv \sqrt{\frac{A_{0}}{A_{2}}},
$$

one obtains the equation

$$
U(1-U)+\frac{d U}{d s}+\frac{1}{c^{2}} \frac{d^{2} U}{(d s)^{2}}+\lambda \sum_{p=3}^{P} \frac{(-1)^{p}}{c^{p}} A_{p} \frac{d^{p} U}{(d s)^{p}}=0
$$

with $\lambda \equiv \sqrt{A_{0} A_{2}}$.

Eq. (17) provides an expansion of the nonlinear differential equation in terms of $1 / c^{p}$ terms with the $1 / c$ term missing. Limiting (7]) to the three first terms $(\lambda \rightarrow 0)$ gives the equation that one obtains [8] for travelling waves in the F-KPP equation

$$
\partial_{t} u(t, x)=\partial_{x}^{2} u(t, x)+u(t, x)(1-u(t, x))
$$

by inserting the form $u(t, x) \equiv U(s=x / c-t)$. Hence in the expansion around the F-KPP equation, the critical velocity gets renormalized from its original value to $c \sim 2$ by the redefinitions (6). We see that adding higher derivatives around $\gamma$ up to rank $P$ leads to a hierarchy of terms in $1 / c^{p}, p \geq 3$ with $c \geq 2$.

Taking $1 / c$ as the small parameter of an expansion we are looking for an iterative solution

$$
h(s)=h_{0}+\frac{1}{c^{2}} h_{2}+\sum_{p \geq 3} \frac{1}{c^{p}} h_{p} \equiv \frac{1}{2}-U(s) .
$$

Inserting it into Eq.(7) translates into a hierarchy of equations

$$
\begin{aligned}
& h_{0}^{\prime}+h_{0}^{2}-1 / 4=0 \\
& h_{2}^{\prime}+2 h_{0} h_{2}+h_{0}^{\prime \prime}=0 \\
& h_{3}^{\prime}+2 h_{0} h_{3}+\lambda A_{3} h_{0}^{\prime \prime \prime}=0 \\
& h_{4}^{\prime}+2 h_{0} h_{4}+h_{2}^{2}+h_{2}^{\prime \prime}-\lambda A_{4} h_{0}^{\prime \prime \prime}=0 \\
& h_{5}^{\prime}+2 h_{0} h_{5}+2 h_{2} h_{3}+h_{3}^{\prime \prime}+\lambda A_{3} h_{2}^{\prime \prime \prime}=0 \cdots,
\end{aligned}
$$

where we have restricted (for sake of simplicity) the hierarchy of equations to $\mathcal{O}\left(1 / c^{6}\right)$. An iterative solution can easily be found with initial conditions being appropriately chosen, i.e. $h_{0}( \pm \infty)= \pm \frac{1}{2}$ and $h_{i \neq 0}( \pm \infty)=h_{i}(0)=0$ for higher orders. Note that if $h(s)$ is solution, also $h\left(s+s_{0}\right)$ is solution.

The system is iteratively but fully solvable. One first solve the only non-linear equation of the hierarchy, obtaining $h_{0}=\frac{1}{2} \operatorname{th}\left(\frac{s}{2}\right)$. Using the property

$$
\frac{d}{d s} h_{n}(s)+2 h_{0} h_{n}(s) \equiv \frac{1}{c h^{2}(s / 2)} \frac{d}{d s}\left[\operatorname{ch}^{2}(s / 2) h_{n}(s)\right],
$$

all the other linear equations reduce to simple integrations of functions defined recursively from the hierarchy.

The solution of the first three terms of the expansion is

$$
U(s)=\frac{1}{1+e^{s}}-\frac{1}{c^{2}} \frac{e^{s}}{\left(1+e^{s}\right)^{2}} \log \frac{\left(1+e^{s}\right)^{2}}{4 e^{s}}+\frac{1}{c^{3}} \lambda A_{3} \frac{e^{s}}{\left(1+e^{s}\right)^{2}}\left(3 \frac{\left(1-e^{s}\right)}{\left(1+e^{s}\right)}+s\right)+\mathcal{O}\left(\frac{1}{c^{4}}\right) .
$$




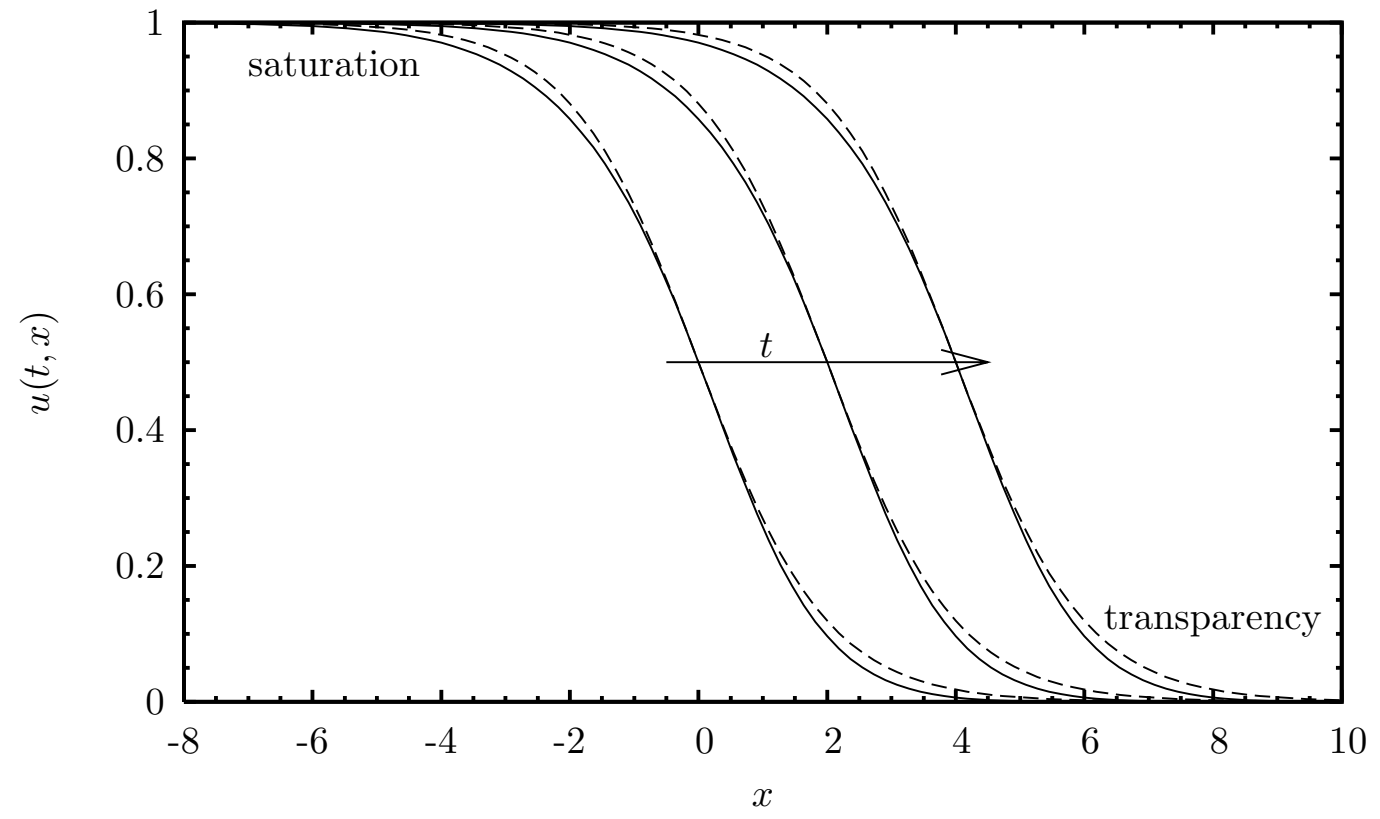

FIG. 1: Universal travelling wave. The function $u(x, t) \equiv U(s=x / c-t)$ is represented in the critical case (c=2) for three different times. Continuous lines: $U(x / 2-t)$; Dashed lines: First $\mathcal{O}\left(1 / c^{0}\right)$ term only. The wave front connecting the regions $u=1$ and $u=0$ travels from the left to the right as $t$ increases. That illustrates how, in terms of QCD properties, the "saturation" region invades the "transparency" region. Note that the first order correction is already small.

This can be compared with the hierarchy of equations obtained for the F-KPP equation (8)

$$
\begin{aligned}
h_{0}^{\prime}+h_{0}^{2}-1 / 4 & =0 \\
h_{2}^{\prime}+2 h_{0} h_{2}+h_{0}^{\prime \prime} & =0 \\
h_{4}^{\prime}+h_{2}^{2}+2 h_{0} h_{4}+h_{2}^{\prime \prime} & =0 \cdots,
\end{aligned}
$$

where the expansion parameter is now $1 / c^{2}$. The solution of the first three equations [8] of (12) is

$$
U(s)=\frac{1}{1+e^{s}}-\frac{1}{c^{2}} \frac{e^{s}}{\left(1+e^{s}\right)^{2}} \log \frac{\left(1+e^{s}\right)^{2}}{4 e^{s}}+\frac{1}{c^{4}}\left\{\frac{e^{s}\left(e^{s}-1\right)}{\left(1+e^{s}\right)^{3}}\left(\frac{1}{2} \log ^{2} \frac{\left(1+e^{s}\right)^{2}}{4 e^{s}}-\log \frac{\left(1+e^{s}\right)^{2}}{4 e^{s}}+3\right)+\frac{s e^{s}}{\left(e^{s}+1\right)^{2}}\right\}+\mathcal{O}\left(\frac{1}{c^{6}}\right) .
$$

The comparison of the sets of equations (9) and (12) and their solutions (11) and (13) calls for comments.

i) The two first terms of the expansion in the small parameter of order 1 and $1 / c^{2}$ are the same, and then give an "universal parametric form" to the travelling wave solution, see Fig 1 The form is unique while the parameters values may change by a redefinition (5) of the coefficients $A_{i}$.

ii) The non-universal dependence on the kernel appears at order $1 / c^{3}$. It can stay small provided the coefficient $A_{3}$ and the followers remain bounded. This implies to keep the expansion of the kernel limited to the region around the chosen value of $\gamma$ and in particular in the vicinity of $\gamma_{c}$ in the critical case $c=2$.

iii) The truncation of the expansion of the kernel is required for the BK equation equipped with the BFKL kernel. Indeed, if considering the full analytic expansion to define the coefficients $A_{i}$ (i.e. $P \rightarrow \infty$ ), they are infinite since they correspond to the expansion of the BFKL kernel near $\gamma=0$ where it possesses a pole singularity. We interpret this phenomenon as a breaking of the travelling wave property in the deep saturation region of the BK equation. In fact, both in the deep saturation region $(u \sim 1$ in Fig.(10) and in the leading edge of the wave $(u \sim 0)$ we expect the exact scaling properties not to be valid [7]. Scaling is expected in between, that is in the "interior" of the wave.

3. Using the redefinitions (6), and limiting ourselves to the universal part, we obtain

$$
\mathcal{N} \propto \frac{1}{1+\left[\frac{k^{2}}{Q_{s}^{2}(Y)}\right]^{\mu_{1}}}-\frac{1}{c^{2}} \frac{\left[\frac{k^{2}}{Q_{s}^{2}(Y)}\right]^{\mu_{1}}}{\left(1+\left[\frac{k^{2}}{Q_{s}^{2}(Y)}\right]^{\mu_{1}}\right)^{2}} \log \frac{\left(1+\left[\frac{k^{2}}{Q_{s}^{2}(Y)}\right]^{\mu_{1}}\right)^{2}}{4\left[k^{2} Q_{s}^{2}(Y)\right]^{\mu_{1}}}+\mathcal{O}\left(1 / c^{3}\right)
$$


where

$$
Q_{s}^{2}(Y)=\exp \left(\mu_{2} Y\right)
$$

plays the role of the saturation scale. In (1415), we have defined $\mu_{1} \equiv \frac{\sqrt{A_{0} / A_{2}}}{c}, \mu_{2} \equiv\left(A_{1}+c\left[\frac{A_{2}}{A_{0}}\right]\right)$. The solution (14) only depends on the function

$$
e^{s} \equiv\left[\frac{k^{2}}{Q_{s}^{2}(Y)}\right]^{\mu_{1}}=\left[\frac{W^{2}}{W_{0}^{2}\left(k^{2}\right)}\right]^{\mu_{2}},
$$

where one writes $W^{2}=e^{Y}, W_{0}=k^{-\mu_{1} / \mu_{2}}$. Hence $e^{s}$ has the typical structure of a forward Regge amplitude. While the first form exhibits the geometric scaling structure, the second one suggest an interpretation of (14) as an unitarization of the amplitude (16).

Hence, we have found the possibility of assessing a specific parametric form to the geometric scaling solutions of the BK equation, through a redefinition of the parameters $\mu_{1}, \mu_{2}$. The form (14), with the possibility of extending the parametrization to higher orders by a solution of the hierarchy (9), may help establishing a link between the theory and the phenomenological observation of geometric scaling [2]. This is the subject of a subsequent work [9].

On a more theoretical level, it seems feasible to extend the method to QCD evolution equations beyond the "mean field" BK equation (1). In particular the extension to the stochastic versions of QCD evolution equations 10 could be treated [9] by averaging the parametric form over the distribution of velocities $c$ generated by the noise.

In conclusion, the fruitful properties of travelling-wave solutions of non-linear evolution equations, both through the asymptotic limit and through their expansion in terms of the inverse velocity give significant tools to deepen our understanding of QCD in the high density limit and emphasize once more the interesting connections with statistical physics.

R.P. wishes to thank C. Marquet and G. Soyez for help and fruitful discussions.

* Electronic address: pesch@spht.saclay.cea.fr

[1] S. Munier and R. Peschanski, Phys. Rev. Lett. 91, 232001 (2003) hep-ph/0309177, Phys. Rev. D 69, 034008 (2004) hep-ph/0310357, Phys. Rev. D 70, 077503 (2004) hep-ph/0401215

[2] A. M. Staśto, K. Golec-Biernat, and J. Kwiecinski, Phys. Rev. Lett. 86, 596 (2001), hep-ph/0007192

[3] I. I. Balitsky, Nucl. Phys. B 463, 99 (1996) hep-ph/9509348 Y. V. Kovchegov, Phys. Rev. D60, 034008 (1999), hep-ph/9901281 Phys. Rev. D61, 074018 (2000), hep-ph/9905214

[4] L. N. Lipatov, Sov. J. Nucl. Phys. 23, 338 (1976); E. A. Kuraev, L. N. Lipatov, and V. S. Fadin, Sov. Phys. JETP 45, 199 (1977); I. I. Balitsky and L. N. Lipatov, Sov. J. Nucl. Phys. 28, 822 (1978).

[5] R. A. Fisher, Ann. Eugenics 7, 355 (1937); A. Kolmogorov, I. Petrovsky, and N. Piscounov, Moscou Univ. Bull. Math. A1, 1 (1937).

[6] M. Bramson, Memoirs of the American Mathematical Society 285 (1983).

[7] U. Ebert, W. van Saarloos, Physica D 146, 1 (2000). For a review, see W. van Saarloos, Phys. Rep. 386, 29 (2003).

[8] J. David Logan, "An Introduction to Nonlinear Partial Differential Equations", John Wiley and sons eds., New York, (1994).

See also P.L. Sachdev, "Self-similarity and beyond, exact solutions of nonlinear problems" Chapman and Hall/CRC, Boca Raton, eds. (2000). The first two terms of the F-KPP solution (13) appear in P.L. Sachdev's book. We thank G.Soyez for a calculation of the third term.

[9] C. Marquet, G. Soyez, R. Peschanski, in preparation.

[10] E. Iancu, A. H. Mueller and S. Munier, Phys. Lett. B 606, 342 (2005) hep-ph/0410018

E. Iancu and D. N. Triantafyllopoulos, hep-ph/0411405 